\title{
EPIS ARE ONTO FOR FINITE REGULAR SEMIGROUPS
}

\author{
by T. E. HALL and P. R. JONES
}

(Received 11th March 1981)

After preliminary results and definitions in Section 1, we show in Section 2 that any finite regular semigroup is saturated, in the sense of Howie and Isbell [8] (that is, the dominion of a finite regular semigroup $U$ in a strictly containing semigroup $S$ is never $S$ ). This is equivalent of course to showing that in the category of semigroups any epi from a finite regular semigroup is in fact onto. Note for inverse semigroups the stronger result, that any inverse semigroup is absolutely closed [11, Theorem VII. 2.14] or [8, Theorem 2.3]. Further, any inverse semigroup is in fact an amalgamation base in the class of semigroups [10], in the sense of [5]. These stronger results are known to be false for finite regular semigroups [8, Theorem 2.9] and [5, Theorem 25]. Whether or not every regular semigroup is saturated is an open problem.

In Section 3 we show that if a regular semigroup has a full subsemigroup (one containing all the idempotents) which is a band of groups, then it has a maximum such subsemigroup. This is to enable us to prove later an amalgamation result for bands of groups.

In Section 4 we show that epis are onto in the categories consisting of the finite members from the following classes, together with all semigroup homomorphisms as morphisms (we note that it is not known if Isbell's Zigzag Theorem also determines dominions in these categories other than that in (i)): (i) regular semigroups; (ii) orthodox semigroups; (iii) unions of groups; (iv) orthodox unions of groups; (v) quasi-orthodox semigroups; (vi) bands of groups; and (vii) orthodox bands of groups.

In Section 5 we show that epis are onto in the category consisting of the finite members from each of the varieties of bands; for the variety of all bands this is due to H. E. Scheiblich [14, Corollary 3.4].

We note at this point that a proof of P. G. Trotter [15, Theorem 1.1] that monos are one-to-one in the category of all regular semigroups is also valid for each of the categories considered in Sections 4 and 5. Hence these categories are balanced, in the usual sense of [13].

\section{Preliminaries}

Let $\mathscr{C}$ be any class of semigroups and take any $U, S \in \mathscr{C}$ with $U$ a subsemigroup of $S$. We say that $U$ dominates an element $d \in S$ within $\mathscr{C}$ if, for every semigroup $W \in \mathscr{C}$ and all homomorphisms

$$
\phi: S \rightarrow W, \quad \psi: S \rightarrow W, \quad \phi(u)=\psi(u) \text { for all } u \in U
$$


implies $\phi(d)=\psi(d)$. The set of all elements of $S$ dominated within $\mathscr{C}$ by $U$ is called the dominion of $U$ in $S$ within $\mathscr{C}$; it is obviously a subsemigroup of $S$ containing $U$. We call $U$ closed in $S$ within $\mathscr{C}$ if the dominion of $U$ in $S$ within $\mathscr{C}$ is just $U$.

A semigroup $U \in \mathscr{C}$ is called absolutely closed within $\mathscr{C}$ if $U$ is closed within $\mathscr{C}$ in every containing semigroup $S \in \mathscr{C}$. A semigroup $U \in \mathscr{C}$ is called saturated within $\mathscr{C}$ if for every semigroup $S \in \mathscr{C}$ having $U$ as a proper subsemigroup, the dominion of $U$ in $S$ within $\mathscr{C}$ is not all of $S$.

When $\mathscr{C}$ is the class of all semigroups we allow ourselves to omit the phrase "within $\mathscr{C} "$ ", and then our definitions are precisely those of Howie and Isbell [8].

We may regard $\mathscr{C}$ as a category by taking morphisms to be all semigroup homomorphisms between members of $\mathscr{C}$. The epis of $\mathscr{C}$ are of course the right cancellable morphisms in $\mathscr{C}$, as in [13].

For any $U, S \in \mathscr{C}$, we say that $U$ is epimorphically embedded in $S$ within $\mathscr{C}$ if $U$ is a subsemigroup of $S$ with dominion equal to $S$ within $\mathscr{C}$. The insertion of $U$ in $S$ is then an epi in the category $\mathscr{C}$; in fact, obviously a morphism $\phi: T \rightarrow S$ in $\mathscr{C}$ is an epi if and only if the dominion of $T \phi$ in $S$ within $\mathscr{C}$ is all of $S$.

The following result is of basic importance.

Result 1. (Isbell's Zigzag Theorem [12, Theorem 2.3] or [11, Theorem VII. 2.13]) Let $U$ be any subsemigroup of any semigroup $S$ and let $d$ be any element of $S$. Then $d$ is in the dominion of $U$ in $S$ if and only if either $d \in U$ or there are elements

$$
u_{1}, v_{1}, u_{2}, v_{2}, \ldots, u_{n}, v_{n}, u_{n+1} \in U, s_{1}, s_{2}, \ldots, s_{n}, t_{1}, t_{2}, \ldots, t_{n} \in S
$$

such that

$$
\begin{gathered}
d=s_{1} u_{1}, \quad u_{1}=v_{1} t_{1}, \quad u_{i+1} t_{i}=v_{i+1} t_{i+1}, \quad s_{i} v_{i}=s_{i+1} u_{i+1}, \\
s_{n} v_{n}=u_{n+1}, \quad i=1,2, \ldots, n-1 .
\end{gathered}
$$

The equations in Result 1 are called a zigzag of length $n$ over $U$ with value $d$, and with spine $u_{1}, v_{1}, u_{2}, v_{2}, \ldots, u_{n}, v_{n}, u_{n+1}$.

We will see below that Isbell's Zigzag Theorem is also valid for $\mathscr{C}$ the class of finite semigroups, of regular semigroups, and of finite regular semigroups (Theorem 2). It is still an open problem whether it is valid for $\mathscr{C}$ the class of bands (raised by Scheiblich [14]).

Result 2. ([1, Proposition 2] or [11, Proposition II. 4.5]) Let $U$ be any regular subsemigroup of any semigroup $S$. For any elements $u, v \in U, u \mathscr{L} v$ if and only if $u \mathscr{L} v$ in $S$.

Result 3. ([3] or [11, Chapter II, Exercise 15]) For any regular semigroup $S$, the subsemigroup $\langle E\rangle$ generated by the set $E$ of idempotents of $S$ is also regular. Further $V\left(E^{n}\right)=E^{n+1}$, for $n=1,2,3, \ldots$

Result 4. [4, Theorem 5] The maximum congruence contained in $\mathscr{H}$ on any regular 
semigroup $S, \mu=\mu(S)$ say, is given by

$$
\begin{gathered}
\mu=\left\{(a, b) \in S \times S: \text { for some inverses } a^{\prime} \text { of } a \text { and } b^{\prime} \text { of } b, a a^{\prime}=b b^{\prime}, a^{\prime} a=b^{\prime} b\right. \\
\text { and } \left.a^{\prime} e a=b^{\prime} e b \text { for each idempotent } e \leqq a a^{\prime}\right\} .
\end{gathered}
$$

Result 5. [4, Corollary 6] Let $U$ be any regular subsemigroup of a regular semigroup $S$ such that $U$ is full in $S$, i.e. contains all the idempotents of $S$. Then $\mu(U)$ $=\mu(S) \cap(U \times U)$.

Result 6. [4, Theorem 14] Let $E$ be any set of idempotents of any semigroup $S$.

(i) There is a regular subsemigroup of $S$ with $E$ as its set of all idempotents if (and only if) $\langle E\rangle$, the subsemigroup generated by $E$, is such a semigroup, i.e., a regular semigroup with $E$ as its set of idempotents.

(ii) If $\langle E\rangle$ is a regular subsemigroup with $E$ as its set of idempotents then

$$
\begin{aligned}
E^{c}=\{a \in S: & \text { for some } a^{\prime} \in V(a), a a^{\prime}, a^{\prime} a, a^{\prime} e a, a f a^{\prime} \in E \text { for all } e, f \in E \text { such that } \\
& \left.e \leqq a a^{\prime}, f \leqq a^{\prime} a\right\}
\end{aligned}
$$

is the maximum regular subsemigroup of $S$ with $E$ as its set of idempotents.

Result 7. Let $U$ be any regular subsemigroup of any regular semigroup $S$. If $U$ contains elements from each $\mathscr{L}$-class and each $\mathscr{R}$-class of $S$ then $U$ is full in $S$.

Proof. Take any idempotent $e \in S$. Then there exist $u, v \in U$ such that $u \mathscr{L} e \mathscr{R} v$, whence $u \mathscr{R} u v \mathscr{L} v$ in $S$ [2, Theorem 2.17] and hence in $U$, by Result 2 and its dual, so again by [2, Theorem 2.17] there is an idempotent $f \in U$ such that $u \mathscr{L} f \mathscr{R} v$, i.e. such that $f \mathscr{H} e$. Hence $e=f \in U$ and $U$ is full in $S$.

We use, whenever possible, and often without comment, the notations and conventions of Clifford and Preston [2] or Howie [11].

\section{Epis from finite regular semigroups}

Theorem 1. Any completely semisimple semigroup with a finite number of $\mathscr{J}$-classes is saturated. In particular, any finite regular semigroup is saturated.

Proof. For the moment, let us just take any completely semisimple semigroup $U$ and any properly containing semigroup $S$ such that the dominion of $U$ in $S$ is all of $S$. Henceforth, the symbol $J_{x}$ shall mean a $\mathscr{J}$-class of $S$ (rather than of $U$ ) and $\mathscr{H}, \mathscr{L}, \mathscr{R}$, $\mathscr{D}, \mathscr{J}$ shall mean Green's relations on $S$.

For any element $s \in S \backslash U$ let $s=s_{1} u_{1}$ and $u_{1}=v_{1} t_{1}$ be the first two equations of a zigzag of least possible length over $U$ with value $s$. Then

$$
J_{u_{1}} \leqq J_{t_{1}} \quad \text { and } \quad u_{1} \in U, \quad t_{1} \in S \backslash U .
$$


Hence the set $\left\{J_{u} \in S / \mathscr{J}: u \in U, J_{u} \leqq J_{t}\right.$ for some $\left.t \in S \backslash U\right\}$ is nonempty. Thus, if $S$ satisfies the ascending chain condition on $\mathscr{J}$-classes, or if $U$ has only finitely many $\mathscr{J}$ classes, there is a maximal member in the above set of $\mathscr{J}$-classes, say $J$.

Take any $J^{\prime} \in S / \mathscr{J}$ such that $J<J^{\prime}$. We show that $J^{\prime} \subseteq U$. Suppose to the contrary that there is an element $s \in J^{\prime} \cap(S \backslash U)$. Then

$$
J<J^{\prime}=J_{s} \leqq J_{u_{1}} \leqq J_{t_{1}}
$$

for some $u_{1} \in U, t_{1} \in S \backslash U$ as above, contradicting the maximality of $J$; hence $J^{\prime} \subseteq U$ as required. It follows also that $J$ contains elements of $S \backslash U$ (since $J \leqq J_{t}$ for some $t \in S \backslash U$ ).

Let $D$ be any $\mathscr{D}$-class (or equivalently $\mathscr{J}$-class) of $U$ contained in $J$. Now $D$ is not a complete $\mathscr{D}$-class of $S$, since otherwise, containing idempotents minimal among its set of idempotents, it would be a complete $\mathscr{J}$-class of $S$ by $[4$, proof of Result 6], a contradiction. Hence there are elements $u \in D, s \in S \backslash U$ such that $u \mathscr{D} s$ in $S$. Thus $u \mathscr{R} t \mathscr{L} S$ for some $t \in S$. Now either $t \in S \backslash U$ or $t \in U \cap R_{u} \subseteq D$ so we have either

$$
u \mathscr{R} t \text { for some } u \in D, \quad t \in S \backslash U
$$

or

$$
t \mathscr{L} S \text { for some } t \in D, \quad s \in S \backslash U \text {. }
$$

Since these two situations are dual to each other, we may assume without loss of generality that $u \mathscr{R} t$ for some $u \in D, t \in S \backslash U$. We show further that $t$ can be taken to be an idempotent. Take any idempotent $e$ in $U$ such that $e \mathscr{R} u$ and let

$$
\begin{gathered}
t=s_{1} u_{1}, \quad u_{1}=v_{1} t_{1}, \quad u_{i+1} t_{i}=v_{i+1} t_{i+1} \\
s_{i} v_{i}=s_{i+1} u_{i+1}, \quad s_{n} v_{n}=u_{n+1}, \quad i=1,2, \ldots, n-1,
\end{gathered}
$$

be any zigzag over $U$ with value $t$ of least possible length, so that $t_{1}, s_{n} \in S \backslash U$. Since $e \mathscr{R} u \mathscr{R} t$ we have $e t=t$ so we can assume without loss of generality that

$$
e s_{i}=s_{i}, \quad e u_{n+1}=u_{n+1}, \quad i=1,2, \ldots, n,
$$

(since otherwise each $s_{i}$ can be replaced by $e s_{i}$, and $u_{n+1}$ by $e u_{n+1}$ ). Since also $t=s_{i} v_{i} t_{i}$ $=u_{n+1} t_{n}$, we have $e \mathscr{R} t \mathscr{R} s_{i} \mathscr{R} u_{n+1}, i=1,2, \ldots, n$.

Now $u_{n+1}=s_{n} v_{n}=s_{n} v_{n} u_{n+1}^{\prime} u_{n+1}=s_{n} \bar{v}_{n} \quad$ where $u_{n+1}^{\prime} \in V\left(u_{n+1}\right) \cap U$ and $\bar{v}_{n}$ $=v_{n} u_{n+1}^{\prime} u_{n+1} \mathscr{L} u_{n+1}$. From $\bar{v}_{n} \mathscr{L} u_{n+1} \mathscr{R} s_{n}$ and $u_{n+1}=s_{n} \bar{v}_{n}$ we have by [2, Theorem 2.17] that $L_{s_{n}} \cap R_{\bar{v}_{n}}$ contains an idempotent, $f$ say.

We show that $f \in S \backslash U$. Suppose to the contrary that $f \in U$. Then $u_{n+1} \mathscr{L}_{\bar{v}} \mathscr{R} f=f^{2}$ so there is a unique element $x \in R_{u_{n+1}} \cap L_{f} \cap U$ such that $x \bar{v}_{n}=u_{n+1}$, by Green's Lemma [11, Lemma II. 2.1] for $U$ and Result 2. Now $s_{n} \in R_{u_{n+1}} \cap L_{f}$ and $s_{n} \bar{v}_{n}=u_{n+1}$, so again by Green's lemma, this time for $S$, we have $s_{n}=x \in U$, a contradiction; thus $f \in S \backslash U$, and $f \mathscr{R} \bar{v}_{n} \in D$, so without loss of generality we may in fact assume that the element $t$ above is an idempotent.

First, from $t^{2}=t \mathscr{R} u_{n+1}$ we have $t u_{n+1}=u_{n+1}$ and from $t=t^{2}=s_{1} u_{1} t$ we have $t \mathscr{L} u_{1} t$, so 
that, since $\mathscr{L}$ is a right congruence on $S$, we also have

$$
u_{1} u_{n+1}=u_{1}\left(t u_{n+1}\right) \mathscr{L} t u_{n+1}=u_{n+1}
$$

Since $u_{1}=v_{1} t_{1}$ and $t_{1} \in S \backslash U$, from

$$
J=J_{t}=J_{s_{1} u_{1}} \leqq J_{u_{1}} \leqq J_{t_{1}}
$$

we have that $J_{u_{1}}=J$, by the maximality of $J$, i.e. $u_{1} \in J$.

So far $D$ has been an arbitrary $\mathscr{J}$-class of $U$ in $J$, but we shall henceforth assume that it is in fact maximal among the $\mathscr{J}$-classes of $U$ in $J$ (let us assume, say, that $U$ satisfies the ascending chain condition on $\mathscr{J}$-classes).

From $u_{1} \in J$ and $u_{1} u_{n+1} \in D\left(u_{1} u_{n+1} \mathscr{L} u_{n+1} \mathscr{R} e\right.$ in $S$ and hence in $\left.U\right)$ we have $u_{1} \in D$ also, by the maximality of $D$. From $u_{1}, u_{n+1}, u_{1} u_{n+1} \in D$ we have that $L_{u_{1}} \cap R_{u_{n+1}} \cap U$ contains an idempotent, $g$ say (since the principal factor $D \cup\{0\}$ of $U$ is of course completely 0 -simple).

From $t=s_{1} u_{1}$ we have $L_{t} \leqq L_{u_{1}}=L_{g}$ whence $t g=t$, and from $t \mathscr{R} u_{n+1} \mathscr{R} g$ we have $t g=g$, giving that $t=g \in U$, a contradiction. Thus the dominion of $U$ in $S$ is not $S$, as required, under the assumption that the completely semisimple semigroup $U$ has only a finite number of its own $\mathscr{J}$-classes, or that both $S$ and $U$ satisfy the ascending chain condition on their own $\mathscr{J}$-classes. This completes the proof.

An amalgam of semigroups is a list $\left(S_{i}, i \in I ; U\right)$ of semigroups such that $U$ is a subsemigroup of $S_{i}, i \in I$. The amalgam $\left(S_{i}, i \in I ; U\right)$ is strongly embeddable in a semigroup $W$ if there exist monomorphisms $\phi_{i}: S_{i} \rightarrow W, i \in I$, such that (i) $\phi_{i}\left|U=\phi_{j}\right| U$ for all $i, j \in I$ and (ii) $\left(S_{i} \phi_{i}\right) \cap\left(S_{j} \phi_{j}\right)=U \phi_{i}$, for all distinct $i, j \in I$.

Theorem 2. Let $\mathscr{C}$ denote any one of the following categories, where morphisms are taken to be semigroup homomorphisms:

(i) finite semigroups;

(ii) regular semigroups;

(iii) finite regular semigroups.

Take any semigroups $U, S$ in $\mathscr{C}$ with $U$ a subsemigroup of $S$. Then the dominion of $U$ in $S$ within $\mathscr{C}$ is the same as within the category of all semigroups.

Proof. We denote by $D$ the dominion of $U$ in $S$ within the category of all semigroups.

(i) From [6, Remark 1] we see that the amalgam $(S, S ; D)$ is strongly embeddable in a finite semigroup (not just a semigroup).

(ii) Since any semigroup is embeddable in a regular semigroup, we have that the amalgam $(S, S ; D)$ is strongly embeddable in a regular semigroup.

(iii) Since any finite semigroup is embeddable in a finite regular semigroup, we have 
from the proof of (i) that the amalgam $(S, S ; D)$ is strongly embeddable in a finite regular semigroup.

It follows that in each case $D$ is also the dominion of $U$ in $S$ within $\mathscr{C}$. From Theorems 1 and 2 we have the following corollaries.

Corollary 3. In the categories of semigroups, regular semigroups and finite semigroups, epis from completely semisimple semigroups with a finite number of $\mathscr{J}$-classes are onto.

Corollary 4. In the category of finite regular semigroups, epis are onto.

Remark 1. The proof of Theorem 1 can be greatly shortened when one assumes that the semigroup $S$ is finite, and such a proof appears in [7]. This weaker result is sufficient to yield Corollary 4 , and also Corollary 3 for the category of finite semigroups.

Example 1. This example, due to Isbell [12, Example 3.1], shows that epis are not onto in the category of finite semigroups. Let $S=\left\{0, e, f, a, a^{-1}\right\}$ be the combinatorial Brandt semigroup with five elements, where $a$ and $a^{-1}$ denote the nonidempotent elements. The subsemigroup $U=\{0, e, f, a\}$ is easily seen to be epimorphically embedded in $S$ within the category of [finite] semigroups.

Whether or not epis are onto in the category of regular semigroups is an open problem.

\section{The maximum full band-of-groups subsemigroup}

For an amalgamation result proved in Section 4 for bands of groups, we require the main result of this section, namely that if a regular semigroup has a full subsemigroup which is a band of groups, then it has a maximum such subsemigroup.

Lemma 5. Let $S$ be any regular semigroup, $E$ its sets of idempotents, and $\mu=\mu(S)$ its maximum idempotent-separating congruence. There is a full band-of-groups subsemigroup of $S$ if and only if $\langle E\rangle$, the subsemigroup generated by $E$, is a band of groups, and this is the case if and only if $S / \mu$ is orthodox. In this case, there is a maximum full band-of-groups subsemigroup of $S$, namely.

$$
\begin{aligned}
\operatorname{ker} \mu & =\cup\{e \mu \in S / \mu: e \in E\} \\
= & \left\{a \in S: \quad \text { for some } a^{\prime} \in V(a), a a^{\prime}=a^{\prime} a \text { and } a^{\prime} e a=e\right. \\
& \text { for each idempotent } \left.e \leqq a a^{\prime}\right\} .
\end{aligned}
$$

Proof. Suppose there is a full band-of-groups subsemigroup $T$ of $S$. The subsemigroup $\langle E\rangle$ of $T$ is regular, by Result 3, and $V_{T}(\langle E\rangle)=\langle E\rangle$ also by Result 3, so $\langle E\rangle$ is a union of groups and hence a band of groups (of course $V_{T}(\langle E\rangle)$ denotes the set of inverses in $T$ of all elements in $\langle E\rangle)$. This proves the first "if and only if" statement.

To prove the second such statement, let us suppose first that $S / \mu$ is orthodox. From Lallement's Lemma [11, Lemma II. 4.6] the band of idempotents of $S / \mu$ is $\{e \mu: e \in E\}$. 
Since $\mu \subseteq \mathscr{H}$, each $e \mu$ is a group and $\{e \mu: e \in E\}$ is a set of disjoint groups, and thus $\cup\{e \mu: e \in E\}=\operatorname{ker} \mu$, for example, is a full band-of-groups subsemigroup of $S$, as required.

Conversely, suppose that $\langle E\rangle$ is a band of groups and let $B$ be any full subsemigroup of $S$ which is also a band of groups; we wish to show that $S / \mu$ is orthodox and that $B \subseteq \operatorname{ker} \mu$. From Result 5 we have that

$$
\mu(B)=\mu(S) \cap(B \times B),
$$

i.e. that the function $\phi: B / \mu(B) \rightarrow S / \mu(S)$ mapping $x \mu(B)$ to $x \mu(S)$, for each $x \in B$, is welldefined and one-to-one, and hence is a monomorphism, and by Lallement's Lemma, is onto a full subsemigroup of $S / \mu(S)$. But $B / \mu(B)$ is a band, so $S / \mu(S)$ is orthodox as required.

Now $(B / \mu(B)) \phi$, the range of $\phi$, is the band of $S / \mu(S)$, namely $\{e \mu \in S / \mu: e \in E\}$. But $(B / \mu(B)) \phi=B \mu(B)^{4} \phi=B \mu(S)^{4}$, whence, for each $b \in B$, we have $b \in b \mu(S) \in\{e \mu(S): e \in E\}$. Thus $B \subseteq \cup\{e \mu(S): e \in E\}=\operatorname{ker} \mu(S)$. We have already seen that $\operatorname{ker} \mu$ is a band of groups, and so it is the maximum full subsemigroup of $S$ which is a band of groups. The alternative description of $\operatorname{ker} \mu$ follows routinely from Result 4.

Corollary 6. Let $S$ be any semigroup and $E$ any set of idempotents of $S$. There is a subsemigroup of $S$ which is a band of groups with $E$ as its set of all idempotents if and only if $\langle E\rangle$ is such a subsemigroup. In this case, there is a maximum such subsemigroup, namely

$$
\begin{gathered}
E^{B}=\left\{a \in S: \quad \text { for some } a^{\prime} \in V(a), a a^{\prime}=a^{\prime} a \in E \text { and } a^{\prime} e a=e\right. \\
\text { for each idempotent } \left.e \leqq a a^{\prime}\right\} .
\end{gathered}
$$

Proof. This follows quite easily from Result 6 and the lemma above.

An analogous result for unions-of-groups subsemigroups is Theorem 10 of [6].

\section{Epis in some categories of finite regular semigroups}

In this section, we will show that epis are onto in certain categories of finite regular semigroups.

We note that (see the appendix in [16] due to Hall) quasi-orthodox semigroups are precisely those regular semigroups such that for all idempotents $e, f, g$ such that $e \mathscr{L} f \mathscr{R} g$ there exists an idempotent $h$ such that $e \mathscr{R} h \mathscr{L} g$.

Theorem 7. Let $\mathscr{C}$ be any one of the following classes of semigroups: (i) regular semigroups; (ii) orthodox semigroups; (iii) unions of groups; (iv) orthodox unions of groups; (v) quasi-orthodox semigroups; (vi) bands of groups; and (vii) orthodox bands of groups.

Let $\left(S_{i}, i \in I ; U\right)$ be any amalgam from $\mathscr{C}$ such that $U^{\prime}$ is full in each $S_{i}, i \in I$. Then the amalgam is strongly embeddable in some semigroup $W$ from $\mathscr{C}$, with finisness being preserved.

Proof. By [6, Theorem 8] the amalgam $\left(S_{i}, i \in I ; U\right)$ is strongly embeddable in a 
regular semigroup $W$ such that $U$ and each $S_{i}$ (or isomorphic copies) are full in $W$, with $W$ finite if $I$ and each $S_{i}$ is finite. Immediately then, for $\mathscr{C}$ one of the classes in (i), (ii) or (v), we have $W \in \mathscr{C}$ and the required result. For $\mathscr{C}$ either of the classes in (iii) or (iv), the result is part of [6, Corollary 12]. For $\mathscr{C}$ either of the classes in (vi) or (vii) the result follows from [6, Theorem 8] and Lemma 5.

Corollary 8. Take any semigroups $U, S$ in $\mathscr{C}$ with $U$ a full subsemigroup of $S$. Then the amalgam $(S, S ; U)$ is strongly embeddable in some semigroup $W$ from $\mathscr{C}$, with finiteness being preserved. In particular $U$ is closed in $S$ within $\mathscr{C}$.

Theorem 9. Let $\mathscr{C}$ be any one of the classes listed above in Theorem 7 and let $\mathscr{F}$ be the category consisting of all the finite semigroups of $\mathscr{C}$ together with all morphisms between these semigroups. Then in $\mathscr{F}$, epis are onto.

Proof. Take any semigroups $U, S$ in $\mathscr{F}$, with $U$ being a proper subsemigroup of $S$. To prove the theorem it suffices to show that the insertion $\iota_{U}: U \rightarrow S$ is not an epi (for if $\alpha: T \rightarrow S$ is an epi and $T \alpha=U$ then $\iota_{U}: U \rightarrow S$ is also an epi). Suppose then, to the contrary, that $U$ is epimorphically embedded in $S$.

Take a maximal $\mathscr{J}$-class of $S$ among those $\mathscr{J}$-classes of $S$ containing elements of $S \backslash U$, $J$ say; then of course for any $\mathscr{J}$-class $J^{\prime}$ of $S$ such that $J^{\prime}>J$ we have $J^{\prime} \subseteq U$. Put

$$
I=\cup\left\{J^{\prime \prime} \in S / \mathscr{J}: J^{\prime \prime} \nsupseteq J\right\},
$$

an ideal of $S$, when nonempty. For the convenience of having $I$ nonempty, let us assume, without loss of generality, that $S=S^{0}$ and $0 \in U$ (since we can adjoin a (further) zero if necessary).

Let $\beta$ denote the canonical morphism from $S$ onto $S / I$. Then $U \beta$ is a proper subsemigroup of $S \beta$ and $U \beta$ is epimorphically embedded in $S \beta$ (i.e. $\iota_{U \beta}: U \beta \rightarrow S \beta$ is an epi in $\mathscr{F}$ ). Thus by replacing $U$ and $S$ by $U \beta$ and $S \beta$ respectively if necessary, we can assume without loss of generality that $J$ is the minimum nonzero $\mathscr{J}$-class of $S$, whence $S \backslash U \subseteq J$.

If $U$ is full in $S$, then by Corollary $8, U$ is closed in $S$ within $\mathscr{F}$ (as well as in $\mathscr{C}$ ), so $\iota_{U}: U \rightarrow S$ is not an epi.

We can assume therefore that $U$ is not full in $S$, so from Result 7 we have that either $U$ does not meet every $\mathscr{L}$-class of $S$ or that $U$ does not meet every $\mathscr{R}$-class of $S$. By duality considerations, we can assume without loss of generality that there is an $\mathscr{L}$-class class $L$ of $S$ such that $U \cap L=\square$. Put

$$
V=\cup\left\{L_{u} \in S / \mathscr{L}: u \in U\right\}
$$

Clearly $V \neq S$, since $V \cap L=\square$. We show that $V$ is a subsemigroup of $S$. Take any $v_{1}, v_{2} \in V$. Then $v_{1} \mathscr{L} u_{1}, v_{2} \mathscr{L} u_{2}$, for some $u_{1}, u_{2} \in U$. If $v_{2} \in U$, then (from $\mathscr{L}$ being a right congruence) we have

$$
v_{1} v_{2} \mathscr{L} u_{1} v_{2} \in U
$$


giving that $v_{1} v_{2} \in V$. If $v_{2} \in V \backslash U \subseteq J$, then

$$
\left.L_{v_{1} v_{2}} \leqq L_{v_{2}} \text { (in } S \text { and in } J \cup\{0\}\right)
$$

so either $v_{1} v_{2}=0 \in U \subseteq V$ or $v_{1} v_{2} \mathscr{L} v_{2} \mathscr{L} u_{2} \in U$ (since $J \cup\{0\}$ is a completely 0-simple ideal of $S$ ), whence $v_{1} v_{2} \in V$. In either case then $v_{1} v_{2} \in V$ so $V$ is a subsemigroup.

We construct now a semigroup $W \in \mathscr{F}$ and two morphisms $\phi, \psi: S \rightarrow W$ which agree only on $V$ (since $U \subseteq V \neq S$ then we will have $l_{U} \phi=l_{U} \psi, \phi \neq \psi$, and $l_{U}$ is not an epi). Take now any two sets $S^{\prime}$ and $S^{\prime \prime}$, each disjoint from $S$ and such that

$$
\begin{gathered}
\left|S^{\prime} \cap S^{\prime \prime}\right|=|V|, \\
\left|S^{\prime} \backslash\left(S^{\prime} \cap S^{\prime \prime}\right)\right|=|S \backslash V|, \\
\left|S^{\prime \prime} \backslash\left(S^{\prime} \cap S^{\prime \prime}\right)\right|=|S \backslash V|
\end{gathered}
$$

whence $\left|S^{\prime}\right|=\left|S^{\prime \prime}\right|=|S|$. Let $\phi: S \rightarrow S^{\prime}$ be a bijection of $S$ upon $S^{\prime}$ such that $V$ is mapped onto $S^{\prime} \cap S^{\prime \prime}$ and likewise let $\psi: S \rightarrow S^{\prime \prime}$ be a bijection of $S$ upon $S^{\prime \prime}$ such that $V$ is mapped onto $\dot{S}^{\prime} \cap S^{\prime \prime}$ but further such that $v \phi=v \psi$ for all $v \in V$. For each $s \in S$, denote $s \phi, s \psi$ by $s^{\prime}, s^{\prime \prime}$ respectively and denote $S^{\prime} \cap S^{\prime \prime}$ by $V^{\prime}$ and by $V^{\prime \prime}$. Note that $v^{\prime}=v^{\prime \prime}$ for all $v \in V$. We make $S^{\prime}$ and $S^{\prime \prime}$ into semigroups isomorphic to $S$ by defining $s^{\prime} t^{\prime}=(s t)^{\prime}$ and $s^{\prime \prime} t^{\prime \prime}=(s t)^{\prime \prime}$, for all $s, t \in S$. Now we put $W=S^{\prime} \cup S^{\prime \prime}$ and we extend the binary operations on $S^{\prime}$ and $S^{\prime \prime}$ (which of course agree on $S^{\prime} \cap S^{\prime \prime}=V^{\prime}=V^{\prime \prime}$ ) to one on $W$ by defining, for all $s, t \in S \backslash V$,

$$
\begin{aligned}
& s^{\prime} t^{\prime \prime}=(s t)^{\prime \prime}, \\
& s^{\prime \prime} t^{\prime}=(s t)^{\prime} .
\end{aligned}
$$

We show now that this binary operation on $W$ is associative. Take any $x, y, z \in W$. If $x, y, z \in S^{\prime}$ or if $x, y, z \in S^{\prime \prime}$ then clearly $(x y) z=x(y z)$. To cover the remaining cases, because of the symmetry between $S^{\prime}$ and $S^{\prime \prime}$ in the construction of the groupoid $W$, we can assume without loss of generality that precisely one of $x, y, z$ is in $S^{\prime \prime} \backslash V^{\prime \prime}$ and that at least one of $x, y, z$ is in $S^{\prime} \backslash V^{\prime}$.

Now there are elements $r, s, t \in S$ such that $x$ is $r^{\prime}$ or $r^{\prime \prime}, y$ is $s^{\prime}$ or $s^{\prime \prime}$ and $z$ is $t^{\prime}$ or $t^{\prime \prime}$, and of course each of $(x y) z$ and $x(y z)$ is one of $(r s t)^{\prime}$ and $(r s t)^{\prime \prime}$. Now if $s t \in V$ then $s t \in V \cap(J \cup\{0\}$ ) (since $s \in S \backslash U \subseteq J$ or $t \in S \backslash U \subseteq J$ ) whence $r s t \in S[V \cap(J \cup\{0\})] \subseteq V$ and $(r s t)^{\prime}=(r s t)^{\prime \prime}$. Hence, if $s t \in V$ we have $(x y) z=x(y z)$.

We may assume now that $s t \in S \backslash V$ (whence $s$ or $t \in S \backslash V$ ), and we now find that entirely routine calculations will complete the verification of associativity, in each of the following cases: Case I, $x \in S^{\prime \prime} \backslash V^{\prime \prime}$; Case II(a), $y \in S^{\prime \prime} \backslash V^{\prime \prime}$ and $z \in S^{\prime} \backslash V^{\prime}$; Case II(b), $y \in S^{\prime \prime} \backslash V^{\prime \prime}$ and $z \in V^{\prime}=V^{\prime \prime}$; Case III, $z \in S^{\prime \prime} \backslash V^{\prime \prime}$.

Thus $W$ is a semigroup. Easy checking also shows that in fact $W \in \mathscr{F}$. Since $\phi: S \rightarrow W$, $\psi: S \rightarrow W$ are distinct morphisms in $\mathscr{F}$ which agree on $U$ we have that $\imath_{U} \phi=l_{U} \psi$ and that $l_{U}$ is not an epi, as required, completing the proof of Theorem 9.

Remark 2. The proof also shows that epis are onto in $\mathscr{F}$ if $\mathscr{F}$ is taken to consist of 
all the completely semisimple semigroups from $\mathscr{C}$ satisfying the ascending chain condition on $\mathscr{J}$-classes.

A class $\mathscr{C}$ of semigroups is said to have the strong amalgamation property if every amalgam of semigroups from $\mathscr{C}$ is strongly embeddable in a semigroup from $\mathscr{C}$, and a class $\mathscr{C}$ of semigroups is said to have the special amalgamation property if each amalgam of the special form $(S, S ; U)$ is strongly embeddable in a semigroup from $\mathscr{C}$.

Theorem 10. The class of finite inverse semigroups has the special amalgamation property.

Proof. We merely have to combine [6, Remark 1] with the last sentence of [5, Section 6] to obtain a proof.

Corollary 11. Epis are onto in the category of finite inverse semigroups.

\section{Epis in some categories of finite bands}

In [14] Scheiblich showed that epis are onto in the category of all bands satisfying the ascending chain condition on $\mathscr{J}$-classes [14, Corollary 3.3] and in the category of all finite bands [14, Corollary 3.4]. We show in this section that his construction "preserves the variety of $S$ " when $U$ meets every $\mathscr{J}$-class of $S$, and thus we obtain the following generalisation of his results.

Theorem 12. Let $\mathscr{V}$ be any variety of bands, let $\mathscr{F}$ be the category of all finite members of $\mathscr{V}$ and let $\mathscr{M}$ be the category of all members of $\mathscr{V}$ satisfying the maximal condition (or equivalently the ascending chain condition) on $\mathscr{J}$-classes. Then in both $\mathscr{F}$ and $\mathscr{M}$, epis are onto.

Proof. Take any $U, S$ in either $\mathscr{F}$ or $\mathscr{M}$ such that $U$ is a proper subband of $S$. Once again, we merely have to show that the injection ${ }_{l_{U}}: U \rightarrow S$ is not an epi, in $\mathscr{F}$ or $\mathscr{M}$. Now if $U$ consists only of rectangular bands it is easily seen that $\mathscr{V}$, $\mathscr{F}$ and $\mathscr{M}=\mathscr{V}$ have the special amalgamation property, whence epis are onto in $\mathscr{F}$ and in $\mathscr{M}$. We assume now that $\mathscr{V}$ does not consist entirely of rectangular bands, with the convenience of having $S^{0} \in \mathscr{V}$ and of having any semilattice as a member of $\mathscr{V}$ also. If $U$ does not meet every $\mathscr{J}$-class of $S$ and if $\mathscr{J}^{4}$ denotes the natural map of $S$ onto $S / \mathscr{F}$, then $U \mathscr{J}^{4}$ is a proper subsemilattice of $S \mathscr{J}^{\xi}=S / \mathscr{J}$ whence $U \mathscr{J}^{\xi}$ is not epimorphically embedded in $S / \mathscr{J}$ within $\mathscr{F}$ or $\mathscr{M}$, which follows trivially from the fact that the class of semilattices has the strong and hence the special amalgamation property [9, proof of Theorem 3.1]; it follows that $U$ is not epimorphically embedded in $S$, so we assume henceforth that $U$ contains elements from every $\mathscr{J}$-class of $S$.

Precisely as in the proof of Theorem 9, we can assume without loss of generality that $S=S^{0}, 0 \in U$ and $S \backslash U \subseteq J$, where $J$ is the minimum non-zero $\mathscr{J}$-class of $S$.

Since $U \neq S$, there is an $\mathscr{R}$-class or an $\mathscr{L}$-class of $S$ not meeting $U$. First we consider the case where some $\mathscr{L}$-class of $S$ does not meet $U$. From the dual of a result proved in the proof of [14, Theorem 3.2] we have that $\rho=l_{S} \cup(\mathscr{L} \cap(J \times J))$ is a congruence on $S$, so, by replacing $U$ and $S$ by $U \rho^{4}$ and $S \rho^{\xi}=S / \rho$ if necessary, we can assume without 
loss of generality that $\mathscr{L} \cap(J \times J)=l_{J}$, so that $J$ is a single $\mathscr{R}$-class of $S$. We now construct $W$ precisely as in the proof of Theorem 9 (replacing $V$ by $U$ ), and acknowledge that it is precisely the band constructed by Scheiblich in proving Theorem 3.2 of $[14]$.

We now show that $W \in \mathscr{V}$. Take any identity, in variables $x_{1}, x_{2}, \ldots, x_{k}$ say, satisfied by $S$, say

$$
w\left(x_{1}, x_{2}, \ldots, x_{k}\right)=\bar{w}\left(x_{1}, x_{2}, \ldots, x_{k}\right)
$$

where $w=w\left(x_{1}, x_{2}, \ldots, x_{k}\right)=x_{i_{1}} x_{i_{2}} \ldots x_{i_{m}}$ and $\bar{w}=\bar{w}\left(x_{1}, x_{2}, \ldots, x_{k}\right)=x_{j_{1}} x_{j_{2}} \ldots x_{j_{n}}$, and take any elements $w_{1}, w_{2}, \ldots, w_{k}$ in $W$; of course we wish to show that $w\left(w_{1}, \ldots, w_{k}\right)$ $=\bar{w}\left(w_{1}, \ldots, w_{k}\right)$. Now there exist $s_{1}, s_{2}, \ldots, s_{k} \in S$ such that $w_{i} \in\left\{s_{i}^{\prime}, s_{i}^{\prime \prime}\right), i=1,2, \ldots, k$, and of course $w\left(s_{1}, \ldots, s_{k}\right)=\bar{w}\left(s_{1}, \ldots, s_{k}\right)=s$, say, and

$$
\left\{w\left(w_{1}, \ldots, w_{k}\right), \bar{w}\left(w_{1}, \ldots, w_{k}\right)\right\} \subseteq\left\{s^{\prime}, s^{\prime \prime}\right\} .
$$

Suppose, by way of contradiction, that $w\left(w_{1}, \ldots, w_{k}\right) \neq \bar{w}\left(w_{1}, \ldots, w_{k}\right)$. By the symmetry between $S^{\prime}$ and $S^{\prime \prime}$ in $W$, we can assume without loss of generality that $w\left(w_{1}, \ldots, w_{k}\right)=s^{\prime}$. Then $\bar{w}\left(w_{1}, \ldots, w_{k}\right)=s^{\prime \prime} \neq s^{\prime}$ and $s \in S \backslash U$.

We show now that the band obtained by adjoining an identity element to a twoelement right zero semigroup is in $\mathscr{V}$, in fact is a subband of $S$.

From $w_{i_{1}} w_{i_{2}} \ldots w_{i_{m}}=s^{\prime} \neq s^{\prime \prime}$ it is easily seen that for some $i_{l}, w_{i_{l}}=s_{i_{l}}^{\prime} \in S^{\prime} \backslash U^{\prime}$ and $w_{i_{l+1}}, \ldots, w_{i_{m}} \in U^{\prime}=U^{\prime \prime}$; we show $l<m$ (i.e. " $w_{i_{l+1}}, \ldots, w_{i_{m}}$ exist"). Since $J$ contains elements of $U$ and $S \backslash U$ it is a nontrivial right zero subsemigroup of $S$, so the words $w$ and $\bar{w}$ end in the same variable; but if $w$ and hence $\bar{w}$ end in $x_{i_{l}}$, then $\bar{w}\left(w_{1}, \ldots, w_{k}\right)=s^{\prime}$ $=w\left(w_{1}, \ldots, w_{k}\right)$, a contradiction, so $l<m$. Put $u=s_{i_{l+1}} \ldots s_{i_{m}} \in U$; in fact $u \in U \backslash(J \cup\{0\})$ since $s=w\left(s_{1}, \ldots, s_{k}\right)=s_{i_{1}} \ldots s_{i_{t}} u \in S \backslash U$. Take any $v \in U \cap J$; then $B=\{u, v u, s\}$ is a band with identity $u$, and with $\{v u, s\}$ a two-element right zero subband, as required.

First, since $S$ is not a rectangular band, we have that $x_{i_{l}}$ occurs in the word $\bar{w}=x_{j_{1}} x_{j_{2}} \ldots x_{j_{n}}$. Second, since $B \leqq S, B$ also satisfies $w=\bar{w}$ and then we easily see that in the word $\bar{w}=x_{j_{1}} x_{j_{2}} \ldots x_{j_{n}}$ only the variables $x_{i_{l+1}}, \ldots, x_{i_{m}}$ can follow the last occurrence of the variable $x_{i}$. It follows that $\bar{w}\left(w_{1}, \ldots, w_{k}\right)=s^{\prime}$, not $s^{\prime \prime}$, a contradiction as required, so $W$ satisfies $w=\bar{w}$, and $U$ is not epimorphically embedded in $S$ in $\mathscr{F}$ nor in $\mathscr{M}$ (in the case where an $\mathscr{L}$-class of $S$ does not meet $U$ ).

Dually, if some $\mathscr{R}$-class of $S$ does not meet $U$ then we have again that $U$ is not epimorphically embedded in $S$ in $\mathscr{F}$ nor in $\mathscr{M}$. Thus epis are onto in $\mathscr{F}$ and in $\mathscr{M}$. This completes the proof.

\section{REFERENCES}

1. L. W. Anderson, R. P. Hunter and R. J. Koch, Some results on stability in semigroups, Trans. Amer. Math. Soc. 117 (1965), 521-529.

2. A. H. Clifford and G. B. Preston, The Algebraic Theory of Semigroups (Math. Surveys No. 7, Amer. Math. Soc., Providence, RI, Vol. I, 1961; Vol. II, 1967). 
3. D. G. Firz-Gerald, On inverses of products of idempotents in regular semigroups, J. Austral. Math. Soc. 13 (1972), 335-337.

4. T. E. HaLl, On regular semigroups, J. Algebra 24 (1973), 1-24.

5. T. E. HALL, Representation extension and amalgamation for semigroups, Quart. J. Math. Oxford (2) 29 (1978), 309-334.

6. T. E. Hall, Amalgamation and inverse and regular semigroups, Trans. Amer. Math. Soc. 246 (1978), 395-406.

7. T. E. Hall, Epimorphisms and dominions, Semigroup Forum 24 (1982), 271-283.

8. J. M. Howie and J. R. Isbell, Epimorphisms and dominions II, J. Algebra 6 (1967), 7-21.

9. J. M. Howie, Commutative semigroup amalgams, J. Austral. Math. Soc. 8 (1968), 609-630.

10. J. M. HowIE, Semigroup amalgams whose cores are inverse semigroups, Quart. J. Math. Oxford (2) 26 (1975), 23-45.

11. J. M. Howie, An Introduction to Semigroup Theory (London Math. Soc. Monographs 7 , Academic Press, 1976).

12. J. R. Isbell, Epimorphisms and dominions, Proceedings of the Conference on Categorical Algebra, La Jolla, 1965 (Lange \& Springer, Berlin, 1966), 232-246.

13. B. Mitchell, Theory of Categories (Academic Press, 1965).

14. H. E. Scheiblich, On epics and dominions of bands, Semigroup Forum 13 (1976), 103-114.

15. P. G. Trotrer, Projectives in inverse semigroups, submitted.

16. M. Yamada, On a certain class of regular semigroups, Proceedings of the Symposium on Regular Semigroups at DeKalb (April 1979), 146-179. 